\title{
Interplay between Quantum Shells and Orientation in Quasifission
}

\author{
A. Wakhle, C. Simenel, ${ }^{*}$ D. J. Hinde, M. Dasgupta, M. Evers, D. H. Luong, R. du Rietz, and E. Williams \\ Department of Nuclear Physics, Research School of Physics and Engineering, Australian National University, \\ Canberra, Australian Capital Territory 2601, Australia \\ (Received 22 June 2014; revised manuscript received 21 August 2014; published 28 October 2014)
}

\begin{abstract}
The quasifission mechanism hinders fusion in heavy systems through breakup within zeptoseconds into two fragments with partial mass equilibration. Its dependence on the structure of both the collision partners and the final fragments is a key question. Our original approach is to combine an experimental measurement of the fragments' mass-angle correlations in ${ }^{40} \mathrm{Ca}+{ }^{238} \mathrm{U}$ with microscopic quantum calculations. We demonstrate an unexpected interplay between the orientation of the prolate deformed ${ }^{238} \mathrm{U}$ with quantum shell effects in the fragments. In particular, calculations show that only collisions with the tip of ${ }^{238} \mathrm{U}$ produce quasifission fragments in the magic $Z=82$ region, while collisions with the side are the only ones that may result in fusion.
\end{abstract}

DOI: 10.1103/PhysRevLett.113.182502

PACS numbers: 24.10.Cn, 25.70.Jj

In the late 1970s, Heusch and collaborators measured fission characteristics in heavy-ion collisions that could not be reconciled with the statistical decay of a compound nucleus [1]. Later, the angular anisotropy of the fission fragments was found to be much larger than that predicted by the statistical model in some reactions [2,3], which was taken as a clear signature for an out-of-equilibrium process.

The origin of these characteristics is understood to be a process known as quasifission. Here the dinuclear system fissions before reaching the stage of an equilibrated compound nucleus [3]. Quasifission thus results in fusion hindrance in reactions forming heavy nuclei [4-6]. In fact, this is by far the dominant mechanism suppressing the formation of superheavy elements. The understanding of this process is thus crucial in order to optimize the formation of new heavy and superheavy nuclei.

Since the discovery of quasifission, important progress has been made thanks to extensive experimental studies [7-24]. Correlations between the mass and the angles of the fragments show that quasifission often takes place before a full rotation of the dinuclear system, that is, with typical contact times between the fragments of 5 to $10 \mathrm{zs}[7,8,17]$. The characteristics of the entrance channel-in particular, the deformation $[10,11,13-15]$ and shell structure [21] of the collision partners as well as the fissility of the system $[19,22]$ and its energy $[15,20]$ — were shown to play an important role. Shell effects could also favor the production of fragments in the vicinity of magic nuclei $[12,15,16,24-26]$.

The complex interplay of all these variables that have been identified by experiments dictates quasifission characteristics and probability, and hence the suppression of fusion. To understand the dynamics at play, in particular the interdependency of these variables, it is necessary to perform theoretical calculations. Classical dynamical models have been developed where the system is described as a viscous fluid evolving through a family of parametrized shapes [27-32]. Despite their ability to reproduce some experimental observables, these approaches require parameters, such as the viscosity, which must be provided externally. One possibility is to extract these parameters directly from microscopic approaches [33,34], for which the only parameters are those describing the interaction between nucleons. Here, we take another approach using a quantum microscopic model to directly investigate quasifission dynamics. Although computationally more demanding, microscopic calculations have the advantage of not constraining the shape of the system during its evolution. The quantum aspect of the model is also crucial for investigating the role of shell effects in the dynamics. The theoretical analysis of the experimental data presented in this Letter is performed with modern microscopic calculations based on the time-dependent Hartree-Fock (TDHF) theory. The latter has recently been successful in describing dynamical processes such as vibration [35-38], fusion [39-45], transfer reactions [46-48], deep-inelastic collisions [49], and quasifission in actinide collisions [50,51] (see Ref. [52] for a review), as well as the dynamics of fission fragments [53].

Mass and angle distributions (MADs) of fission fragments formed in ${ }^{40} \mathrm{Ca}+{ }^{238} \mathrm{U}$ collisions have been measured and calculated at different energies to investigate the role of quantum shell effects on the final characteristics of the fragments. In particular, we answer key questions, such as the interplay between the orientation of a deformed collision partner and the quantum shells affecting the outcome of the quasifission reaction. The findings are relevant to understanding the dynamics for forming the next superheavy elements since all the available targets that are planned to be used are deformed.

Pulsed beams of ${ }^{40} \mathrm{Ca}$ were produced using a 14UD electrostatic accelerator followed by a LINAC postaccelerator at the Australian National University. Isotopically enriched targets of ${ }^{238} \mathrm{UF}_{4}\left(250 \mu \mathrm{g} / \mathrm{cm}^{2}\right)$, evaporated onto 
$\sim 15 \mu \mathrm{g} / \mathrm{cm}^{2}{ }^{\text {nat }} \mathrm{C}$ backings, were mounted on a target ladder whose normal was at $60^{\circ}$ to the beam. Binary reaction products were detected in coincidence using two $28 \times 36 \mathrm{~cm}^{2}$ position-sensitive multiwire proportional counters on opposite sides of the beam, covering laboratory scattering angles of $5^{\circ}<\theta<80^{\circ}$ and $50^{\circ}<\theta<125^{\circ}$. The measured positions and times of flight allowed direct reconstruction of the fragment velocities [11]. The latter were converted into mass ratio $M_{R}=m_{1} /\left(m_{1}+m_{2}\right)$ and center-of-mass (c.m.) scattering angle $\theta_{\text {c.m. }}$. for events where only two primary fragments with masses $m_{1}$ and $m_{2}$ are formed. The selection of full momentum transfer (binary) events is described in details in Ref. [22]. Since both fragments are detected, the MAD is populated twice [54], at $\left(M_{R}, \theta_{\text {c.m. }}\right)$ and $\left(1-M_{R}, \pi-\theta_{\text {c.m. }}\right)$.

The MAD measured at three energies are shown in Figs. 1(a)-(c). The azimuthal coincidence coverage of the detector system was $90^{\circ}$ for all $\theta_{\text {c.m. }}$; thus, the number of events in each MAD bin is proportional to the angular differential cross section $d \sigma / d \theta_{\text {c.m. }}$. No events were detected at the most forward and backward angles due to detector angular acceptance. The intense bands at extreme $M_{R}$ values correspond to (quasi)elastic scattering. Events associated with quasifission and fusion fission are located between these two bands.

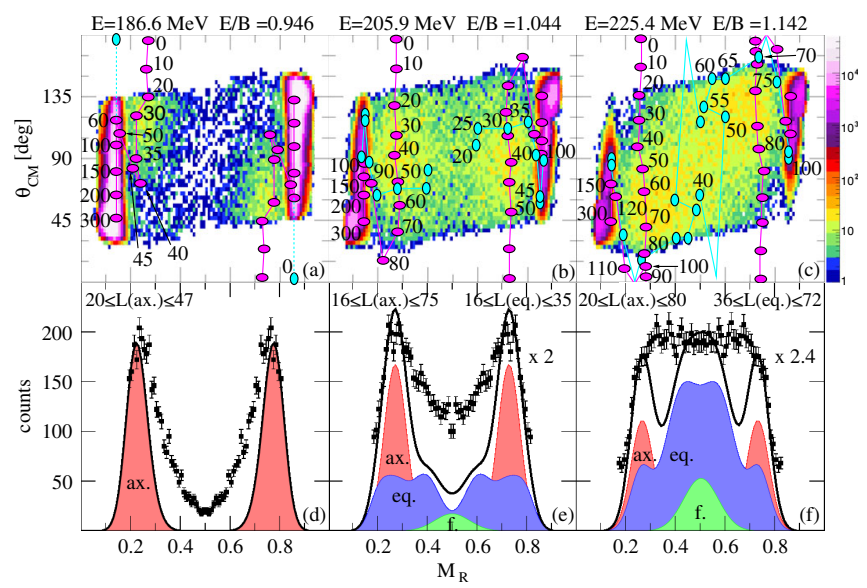

FIG. 1 (color online). (a)-(c) Measured mass-angle distributions at center-of-mass energies $E$ near the capture barrier $B$ [55]. The logarithmic color scale is shown in the top right. The horizontal and vertical ellipsoids show the TDHF results for the axial and equatorial configurations, respectively. The values of $L$ are indicated near the points associated to the light (heavy) fragments for the axial (equatorial) orientation. (d)-(f) Projected mass ratio $M_{R}$ in the range $0.2<M_{R}<0.8$. The scale factor in panels (e) and (f) multiplies the counts scale on the left. The mass ratio distributions estimated from the TDHF results are shown with shaded areas for quasifission in the axial (ax.) and equatorial (eq.) configurations, and for fusion-fission (f.) events. The ranges of $L$ used to calculate the mass ratio distributions are given in panels (d)-(f) and correspond to events falling into the angular acceptance of the detector. The sum of these distributions is shown (solid line).
Each MAD shows very mass-asymmetric groups of fission events, with the light fragment in the range $M_{R} \simeq$ $0.2-0.3$ and a corresponding heavy fragment. These groups move toward forward and backward angles, respectively, with increasing energy. This correlation of mass with angle is a clear signature for quasifission events. In addition, the fraction of events around $M_{R}=0.5$ (indicating mass equilibration of the fragments) increases with energy. These mass symmetric events are compatible with long lifetime quasifission (i.e., with contact times greater than half a rotation), or fusion followed by statistical fission.

The projections of the MADs onto the $M_{R}$ axis are shown with filled squares in Figs. 1(d)-(e). Peaks at $M_{R} \simeq$ 0.25 and 0.75 , associated with asymmetric quasifission, are clearly visible at the two lowest energies. At the highest energy, less asymmetric events dominate and a wide plateau is observed between $M_{R} \simeq 0.3$ and 0.7 , in excellent agreement with mass distributions presented in [20].

To interpret these observations, TDHF calculations were performed at the same energies using the TDHF3D code [56]. The system is described by an antisymmetrized independent particle state at all time to ensure an exact treatment of the Pauli principle, which is crucial at low energies. The TDHF equation is $i \hbar(d / d t) \rho=[h[\rho], \rho]$, where $\rho$ is the one-body density matrix [57]. The Hartree-Fock (HF) Hamiltonian $h[\rho]$ is obtained from a Skyrme energy density functional [58]. Unlike early calculations of similar reactions that used simplified interactions [59], the SLy4d parametrization [56] used here includes a spin-orbit interaction [60]. The latter is crucial for reproducing the one-body dissipative mechanisms [61] that strongly affect low-energy dynamics, as well as magic numbers in heavy nuclei. More details can be found in Ref. [52].

Examples of shape evolution for ${ }^{40} \mathrm{Ca}+{ }^{238} \mathrm{U}$ at $E=225.4 \mathrm{MeV}$ are shown in Fig. 2 for two extreme orientations of the prolate ${ }^{238} \mathrm{U}$ nucleus. In the collision with the tip of ${ }^{238} \mathrm{U}$ (axial orientation) at an angular momentum quantum number $L=100$ (upper panels), the system undergoes half a rotation in $\sim 5 \mathrm{zs}$ before it separates into primary fragments with smaller mass asymmetry. This relatively short contact time and the partial mass equilibration are clear signatures of a

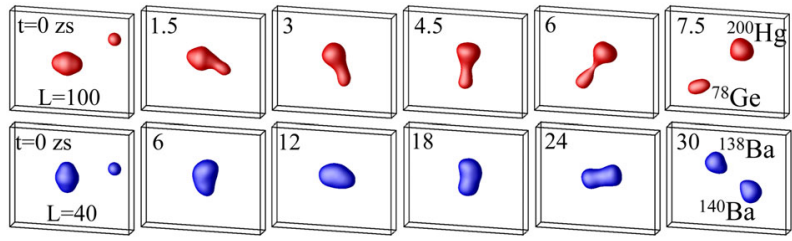

FIG. 2 (color online). Time evolution of the density in ${ }^{40} \mathrm{Ca}+{ }^{238} \mathrm{U}$ collisions at a center-of-mass energy $E=$ $225.4 \mathrm{MeV}$. The isodensity at half the saturation density $\rho_{0} / 2=$ $0.08 \mathrm{fm}^{-3}$ is plotted every $1.5 \mathrm{zs}$ for the axial orientation at $L=100$ (top) and every $6 \mathrm{zs}$ for the equatorial orientation at $L=40$ (bottom). 
quasifission process. The collision with the side of ${ }^{238} \mathrm{U}$ (equatorial orientation) at $L=40$ (lower panels) exhibits a much longer contact time (about $30 \mathrm{zs}$ ) and a full rotation before separation, which is also compatible with a quasifission process. However, this time is long enough to induce a full mass equilibration, leading to symmetric mass split. The total kinetic energy of the final fragments is predicted to be $\sim 243 \mathrm{MeV}$, in excellent agreement with experimental data for $M_{R} \simeq 0.5$ [20].

Systematic calculations have been performed by varying $L$ with steps $\Delta L=10$ or 5. See Supplemental Material [62] for movies of the density evolution associated with these calculations. The results are reported on the MADs in Fig. 1. Axial (equatorial) orientations are represented by purple horizontal (blue vertical) ellipsoids. At the lowest energy [Fig. 1(a)], axial collisions lead to quasifission up to $L \sim 45$, and above that to quasielastic scattering. Equatorial orientations contribute only to the elastic peak. The light quasifission fragments are essentially located at backward angles with mass ratio in the range $M_{R} \sim 0.22-0.27$, in good agreement with the data falling into the angular acceptance of the detector. Similar conclusions can be drawn at higher energies [Figs 1(b) and 1(c)] for the axial collisions, with the angles of the light quasifission fragments going toward more forward angles with increasing energy. Fusion does not occur for the axial orientation. In contrast, equatorial collisions form a compact system that has not decayed into fission fragments at the end of the calculations for $L \leq 10$ at $E=205.9 \mathrm{MeV}$ and $L \leq 30$ at $E=225.4 \mathrm{MeV}$. These events, associated with long lifetime quasifission or with fusion fission, are expected to produce fragments with isotropic angular distributions. They are called "fusion-fission" hereafter. Quasifission events are obtained at larger angular momenta and include more symmetric events than found in axial collisions. These comparisons between TDHF predictions and data are meaningful only when fission of the heavy fragment is negligible. Only fragments significantly heavier than $\mathrm{Pb}$ could fission, affecting the $M_{R} \geq 0.76$ and $M_{R} \leq 0.24$ regions. However, the calculations predict negligible yields of quasifission fragments in this $M_{R}$ region.

In order to estimate the overall significance of the orientation of the ${ }^{238} \mathrm{U}$ nucleus, a representation of the MAD of quasifission fragments is given in Figs. 3(a)-(d) at the two highest energies. Since TDHF calculations underestimate the fluctuation of the fragment mass distributions in damped collisions [63], Gaussian distributions centered around each $M_{R}$ and $\theta_{\text {c.m. }}$ obtained from TDHF calculations and weighted by $2 L+1$ are assumed, with a standard deviation in mass ratio varying linearly with $M_{R}$ from 0.025 at the initial mass split to 0.07 at symmetry [17], and a standard deviation in angle of $20^{\circ}$. The relative weight between the two orientations is determined by assuming that axial collisions are obtained when the angle between the beam axis and the target nucleus symmetry axis is smaller

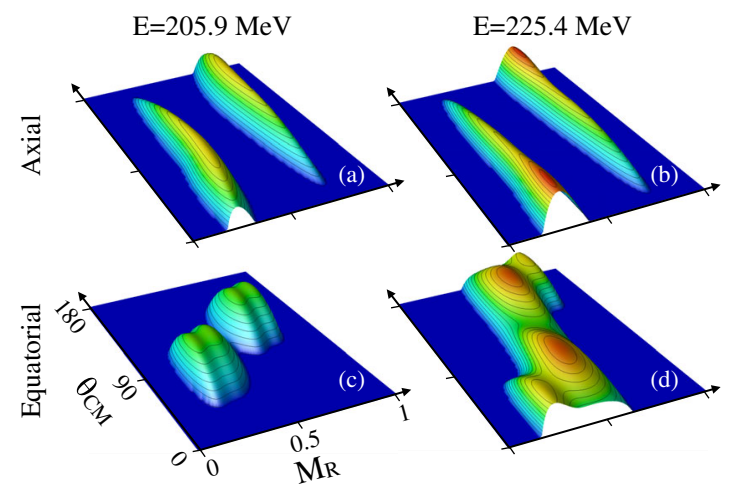

FIG. 3 (color online). Mass-angle distributions of quasifission fragments from TDHF calculations. The common vertical linear scale corresponds to the angular differential cross section in arbitrary units.

than $35^{\circ}$ [11]. The projections on the mass-ratio axis (for the angular acceptance of the detectors) are shown in Figs. 1(d)-(f) by shaded areas, together with fusion-fission events that are assumed to produce symmetric fragments with isotropic angular distributions. The resulting total distributions are normalized to the most mass-asymmetric experimental events. Note that a quantitative reproduction of the experimental MAD is beyond the scope of this work, as it would require extensive calculations at intermediate orientations. It is observed that the axial orientation is mostly responsible for asymmetric quasifission at all energies. The more symmetric events are populated by the equatorial collisions, which are dominant at the highest energy, giving rise to the observed plateau in Fig. 1(f).

To test the validity of this approach, we computed the ratio of the fusion to capture cross sections $\sigma_{f} / \sigma_{c}=0.09 \pm$ 0.07 at $205.9 \mathrm{MeV}$ and $0.16 \pm 0.06$ at $225.4 \mathrm{MeV}$ (no fusion is observed at the lowest energy). The uncertainty is due to the angular momentum mesh. These results are in good agreement with Ref. [8] where a ratio $0.11 \sim 0.06$ was obtained in this energy range. The TDHF capture cross sections at these energies agree with those of Ref. [8] within the $20 \%$ experimental error bars.

We now investigate the role of shell effects in the formation of the fragments. The extra binding energy from shell effects is expected to favor the formation of fragments with magic numbers. This is supported by the massasymmetric quasifission observed in the experimental MAD that has its heavy fragment in the ${ }^{208} \mathrm{~Pb}$ region. The proton numbers of the heavy fragment from TDHF calculations are plotted as a function $L$ in Fig. 4(a). Quasifission in axial collisions (filled symbols) always forms a fragment close to the magic proton number $Z=82$. We observed a similar behavior for neutrons with $N \sim 122$, indicating that the influence of the magic number $N=126$ is not as strong as $Z=82$. These observations do not depend on beam energy. However, for equatorial collisions these magic numbers have no visible effects on the outcomes. 


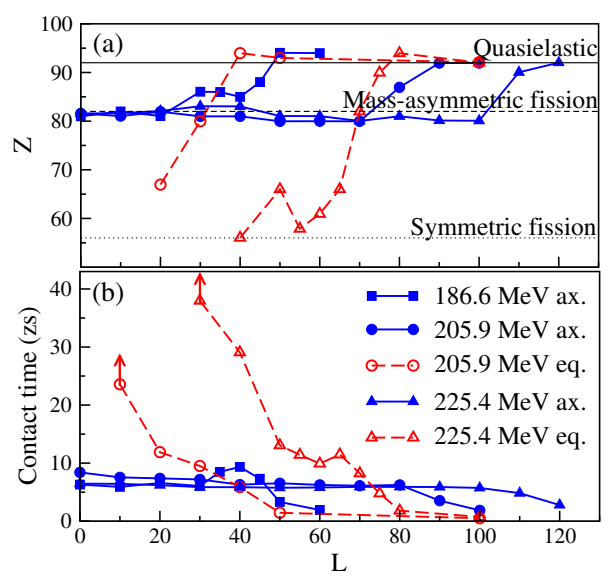

FIG. 4 (color online). (a) Mean number of protons $Z$ in the outgoing heavy fragment as a function of the angular momentum quantum number $L$. Axial and equatorial orientations are represented by thick solid and thick dashed lines, respectively. The horizontal thin solid, thin dashed, and thin dotted lines show the values of $Z$ of the initial ${ }^{238} \mathrm{U}$ nucleus (quasielastic scattering), of the doubly magic ${ }^{208} \mathrm{~Pb}$ nucleus (mass-asymmetric fission), and of the ${ }^{139} \mathrm{Ba}$ fragment formed in mass-symmetric fission, respectively. (b) Contact time between the fragments defined as the time during which the density in the neck exceeds half the saturation density $\rho_{0} / 2=0.08 \mathrm{fm}^{-3}$. The arrows indicate lower limits of the contact time.

One possible reason is that, unlike the axial orientation, equatorial collisions form systems that are more compact than two touching ${ }^{208} \mathrm{~Pb}$ and ${ }^{70} \mathrm{Zn}$ fragments. As shown in Fig. 4(b), the difference in nucleon transfer between the orientations translates into different quasifission times. Indeed, the quasifission times are smaller than $10 \mathrm{zs}$ and are almost independent of $E$ and $L$ for the axial orientation, while quasifission times over $30 \mathrm{zs}$ (see also Fig. 2) are observed for the equatorial orientation.

Experimental mass-angle distributions of quasifission fragments formed in ${ }^{40} \mathrm{Ca}+{ }^{238} \mathrm{U}$ have been measured and interpreted using TDHF calculations. The angular focusing of fragments with large mass asymmetry indicates that they are produced by quasifission. This asymmetric quasifission is related to shell effects in the $Z=82$ region and occurs in collisions with the tip of the ${ }^{238} \mathrm{U}$, leading to short quasifission times. No quantum shell effects were observed in collisions with the side of ${ }^{238} \mathrm{U}$. Long contact times compatible with fusion are found only for this orientation. This first evidence for the orientation dependence of shell effects in quasifission requires further investigation.

Discussions with A.S. Umar and V.E. Oberacker are acknowledged. This work has been supported by the Australian Research Council Grants No. FT120100760, No. FL110100098, and No. DP1094947. The calculations have been performed on the NCI National Facility in Canberra, Australia, which is supported by the Australian Commonwealth Government.
*Corresponding author.

cedric.simenel@anu.edu.au

[1] B. Heusch, C. Volant, H. Freiesleben, R. Chestnut, K. Hildenbrand, F. Pühlhofer, W. Schneider, B. Kohlmeyer, and W. Pfeffer, Z. Phys. A 288, 391 (1978).

[2] B. B. Back, H. G. Clerc, R. R. Betts, B. G. Glagola, and B. D. Wilkins, Phys. Rev. Lett. 46, 1068 (1981).

[3] R. Bock, Y. T. Chu, M. Dakowski, A. Gobbi, E. Grosse, A. Olmi, H. Sann, D. Schwalm, U. Lynen, W. Müller, S. Børnholm, H. Esbensen, W. Wölfli, and E. Morenzoni, Nucl. Phys. A388, 334 (1982).

[4] C.-C. Sahm, H.-G. Clerc, K.-H. Schmidt, W. Reisdorf, P. Armbruster, F. Hessberger, J. Keller, G. Münzenberg, and D. Vermeulen, Z. Phys. A 319, 113 (1984).

[5] H. Gäggeler, T. Sikkeland, G. Wirth, W. Brüchle, W. Bögl, G. Franz, G. Herrmann, J. V. Kratz, M. Schädel, K. Sümmerer, and W. Weber, Z. Phys. A 316, 291 (1984).

[6] K. H. Schmidt and W. Morawek, Rep. Prog. Phys. 54, 949 (1991).

[7] J. Töke, R. Bock, G. Dai, A. Gobbi, S. Gralla, K. Hildenbrand, J. Kuzminski, W. Müller, A. Olmi, H. Stelzer, B. Back, and S. Bjørnholm, Nucl. Phys. A440, 327 (1985).

[8] W. Q. Shen, J. Albinski, A. Gobbi, S. Gralla, K. D. Hildenbrand, N. Herrmann, J. Kuzminski, W. F. J. Müller, H. Stelzer, J. Töke, B. B. Back, S. Bjørnholm, and S. P. Sørensen, Phys. Rev. C 36, 115 (1987).

[9] D. J. Hinde, D. Hilscher, H. Rossner, B. Gebauer, M. Lehmann, and M. Wilpert, Phys. Rev. C 45, 1229 (1992).

[10] D. J. Hinde, M. Dasgupta, J. R. Leigh, J. P. Lestone, J. C. Mein, C. R. Morton, J. O. Newton, and H. Timmers, Phys. Rev. Lett. 74, 1295 (1995).

[11] D. J. Hinde, M. Dasgupta, J. R. Leigh, J. C. Mein, C. R. Morton, J. O. Newton, and H. Timmers, Phys. Rev. C 53, 1290 (1996).

[12] M. Itkis et al., Nucl. Phys. A734, 136 (2004).

[13] G. N. Knyazheva et al., Phys. Rev. C 75, 064602 (2007).

[14] D. J. Hinde, R. G. Thomas, R. du Rietz, A. Diaz-Torres, M. Dasgupta, M. L. Brown, M. Evers, L. R. Gasques, R. Rafiei, and M. D. Rodriguez, Phys. Rev. Lett. 100, 202701 (2008).

[15] K. Nishio, H. Ikezoe, S. Mitsuoka, I. Nishinaka, Y. Nagame, Y. Watanabe, T. Ohtsuki, K. Hirose, and S. Hofmann, Phys. Rev. C 77, 064607 (2008).

[16] E. Kozulin et al., Phys. Lett. B 686, 227 (2010).

[17] R. du Rietz, D. J. Hinde, M. Dasgupta, R. G. Thomas, L. R. Gasques, M. Evers, N. Lobanov, and A. Wakhle, Phys. Rev. Lett. 106, 052701 (2011).

[18] I. M. Itkis, E. M. Kozulin, M. G. Itkis, G. N. Knyazheva, A. A. Bogachev, E. V. Chernysheva, L. Krupa, Y. T. Oganessian, V. I.Zagrebaev, A. Y. Rusanov, F. Goennenwein, O. Dorvaux, L. Stuttgé, F. Hanappe, E. Vardaci, and E. de Goés Brennand, Phys. Rev. C 83, 064613 (2011).

[19] C. J. Lin, R. du Rietz, D. J. Hinde, M. Dasgupta, R. G. Thomas, M. L. Brown, M. Evers, L. R. Gasques, and M. D. Rodriguez, Phys. Rev. C 85, 014611 (2012).

[20] K. Nishio, S. Mitsuoka, I. Nishinaka, H. Makii, Y. Wakabayashi, H. Ikezoe, K. Hirose, T. Ohtsuki, Y. Aritomo, and S. Hofmann, Phys. Rev. C 86, 034608 (2012).

[21] C. Simenel, D. J. Hinde, R. du Rietz, M. Dasgupta, M. Evers, C. J. Lin, D. H. Luong, and A. Wakhle, Phys. Lett. B 710, 607 (2012). 
[22] R. du Rietz, E. Williams, D. J. Hinde, M. Dasgupta, M. Evers, C. J. Lin, D. H. Luong, C. Simenel, and A. Wakhle, Phys. Rev. C 88, 054618 (2013).

[23] E. Williams, D. J. Hinde, M. Dasgupta, R. du Rietz, I. P. Carter, M. Evers, D. H. Luong, S. D. McNeil, D. C. Rafferty, K. Ramachandran, and A. Wakhle, Phys. Rev. C 88, 034611 (2013).

[24] E. M. Kozulin, G. N. Knyazheva, S. N. Dmitriev, I. M. Itkis, M. G. Itkis, T. A. Loktev, K. V. Novikov, A. N. Baranov, W. H. Trzaska, E. Vardaci, S. Heinz, O. Beliuskina, and S. V. Khlebnikov, Phys. Rev. C 89, 014614 (2014).

[25] M. Morjean et al., Phys. Rev. Lett. 101, 072701 (2008).

[26] M. O. Frégeau et al., Phys. Rev. Lett. 108, 122701 (2012).

[27] A. Diaz-Torres, G. G. Adamian, N. V. Antonenko, and W. Scheid, Phys. Rev. C 64, 024604 (2001).

[28] G. G. Adamian, N. V. Antonenko, and W. Scheid, Phys. Rev. C 68, 034601 (2003).

[29] V. Zagrebaev and W. Greiner, J. Phys. G 31, 825 (2005).

[30] Y. Aritomo, Phys. Rev. C 80, 064604 (2009).

[31] M. Huang, Z. Gan, X. Zhou, J. Li, and W. Scheid, Phys. Rev. C 82, 044614 (2010).

[32] Y. Aritomo, K. Hagino, K. Nishio, and S. Chiba, Phys. Rev. C 85, 044614 (2012).

[33] K. Washiyama and D. Lacroix, Int. J. Mod. Phys. E 18, 2114 (2009).

[34] K. Wen, F. Sakata, Z.-X. Li, X.-Z. Wu, Y.-X. Zhang, and S.-G. Zhou, Phys. Rev. Lett. 111, 012501 (2013).

[35] C. Simenel and P. Chomaz, Phys. Rev. C 68, 024302 (2003).

[36] J. A. Maruhn, P. G. Reinhard, P. D. Stevenson, J. R. Stone, and M. R. Strayer, Phys. Rev. C 71, 064328 (2005).

[37] T. Nakatsukasa and K. Yabana, Phys. Rev. C 71, 024301 (2005).

[38] B. Avez and C. Simenel, Eur. Phys. J. A 49, 76 (2013).

[39] C. Simenel, P. Chomaz, and G. de France, Phys. Rev. Lett. 86, 2971 (2001).

[40] A. S. Umar and V. E. Oberacker, Phys. Rev. C 73, 054607 (2006).

[41] K. Washiyama and D. Lacroix, Phys. Rev. C 78, 024610 (2008).

[42] L. Guo and T. Nakatsukasa, EPJ Web Conf. 38, 09003 (2012).
[43] C. Simenel, R. Keser, A. S. Umar, and V. E. Oberacker, Phys. Rev. C 88, 024617 (2013).

[44] C. Simenel, M. Dasgupta, D. J. Hinde, and E. Williams, Phys. Rev. C 88, 064604 (2013).

[45] A. S. Umar, C. Simenel, and V. E. Oberacker, Phys. Rev. C 89, 034611 (2014).

[46] C. Simenel, Phys. Rev. Lett. 105, 192701 (2010).

[47] G. Scamps and D. Lacroix, Phys. Rev. C 87, 014605 (2013).

[48] K. Sekizawa and K. Yabana, Phys. Rev. C 88, 014614 (2013).

[49] C. Simenel, Phys. Rev. Lett. 106, 112502 (2011).

[50] C. Golabek and C. Simenel, Phys. Rev. Lett. 103, 042701 (2009).

[51] D. J. Kedziora and C. Simenel, Phys. Rev. C 81, 044613 (2010).

[52] C. Simenel, Eur. Phys. J. A 48, 152 (2012).

[53] C. Simenel and A.S. Umar, Phys. Rev. C 89, 031601 (2014).

[54] R. G. Thomas, D. J. Hinde, D. Duniec, F. Zenke, M. Dasgupta, M. L. Brown, M. Evers, L. R. Gasques, M. D. Rodriguez, and A. Diaz-Torres, Phys. Rev. C 77, 034610 (2008).

[55] W. J. Świątecki, K. Siwek-Wilczyńska, and J. Wilczyński, Phys. Rev. C 71, 014602 (2005).

[56] K.-H. Kim, T. Otsuka, and P. Bonche, J. Phys. G 23, 1267 (1997).

[57] P. A. M. Dirac, Proc. Cambridge Philos. Soc. 26, 376 (1930).

[58] T. Skyrme, Philos. Mag. 1, 1043 (1956).

[59] H. Stócker, R. Cusson, H. Lustig, A. Gobbi, J. Hahn, J. Maruhn, and W. Greiner, Z. Phys. A 306, 235 (1982).

[60] E. Chabanat, P. Bonche, P. Haensel, J. Meyer, and R. Schaeffer, Nucl. Phys. A635, 231 (1998).

[61] A. S. Umar, M. R. Strayer, and P. G. Reinhard, Phys. Rev. Lett. 56, 2793 (1986).

[62] See Supplemental Material at http://link.aps.org/ supplemental/10.1103/PhysRevLett.113.182502 for movies of the density evolution associated with TDHF calculation.

[63] C. H. Dasso, T. Døssing, and H. C. Pauli, Z. Phys. A 289, 395 (1979). 